\title{
Les bracelets protohistoriques en verre de Bretagne
}

The protohistoric glass bracelets of Brittany

Maryse Dinard, Bernard Gratuze et Anne-Françoise Cherel

\section{OpenEdition}

\section{Journals}

Édition électronique

URL : http://journals.openedition.org/rao/1566

DOI : $10.4000 /$ rao. 1566

ISBN : 978-2-7535-1846-9

ISSN : $1775-3732$

\section{Éditeur}

Presses universitaires de Rennes

\section{Édition imprimée}

Date de publication : 31 décembre 2011

Pagination : 149-166

ISBN : 978-2-7535-1844-5

ISSN : 0767-709X

\section{Référence électronique}

Maryse Dinard, Bernard Gratuze et Anne-Françoise Cherel, « Les bracelets protohistoriques en verre de Bretagne », Revue archéologique de l'Ouest [En ligne], 28 | 2011, mis en ligne le 30 mars 2014, consulté le 05 décembre 2020. URL : http://journals.openedition.org/rao/1566 ; DOI : https://doi.org/10.4000/ rao. 1566 


\title{
Les bracelets protohistoriques en verre de Bretagne

\author{
The protohistoric glass bracelets of Brittany
}

\author{
Maryse DinarD* \\ avec la collaboration de Bernard Gratuze ${ }^{* *}$ et Anne-Françoise Cherel ***
}

Résumé : D'après les sources bibliographiques et des échanges directs avec des archéologues, les bracelets protohistoriques en verre de Bretagne ont pu être recensés. Dans le corpus des 43 objets ainsi répertoriés, certains ont pu être associés aux typologies existantes (Haevernick et Gebhard). Les autres n'y trouvant pas de correspondances, il nous a fallu mettre en place un nouveau codage typologique. Présenté ici, il prend en compte à la fois la forme de la section, la couleur et le décor. 17 de ces bracelets ont également bénéficié d'analyses chimiques, ce qui a permis de déterminer l'origine probable de leurs matières premières. De plus, ces résultats ouvrent de nouvelles perspectives de recherche.

\begin{abstract}
From bibliographic sources and correspondence with archaeologists, the protohistoric glass bracelets of Brittany have been listed. In this corpus of 43 examples, some could be related to existing typologies (Hevernick and Gebhard). As the others had no connexion with these, a new typological scheme - presented here - had to be devised by shape of section, color and decoration. 17 of these bracelets were also chemcally analysed to determine the raw materials' probable origin. These results provide new research prospects.
\end{abstract}

Mots clés : parure, verre, second âge du Fer, Bretagne, typologie, analyses ICP-MS, datation, Orient, atelier.

Key words: jewellery, glass, Second Iron Age, Brittany, typology, ICP-MS analysis, dating, East, workshop.

\section{INTRODUCTION}

Les récentes études menées par Anne-Françoise Cherel et Bernard Gratuze sur les perles protohistoriques en verre de Bretagne ${ }^{1}$ ont soulevé la nécessité de faire le point sur nos connaissances sur les bracelets protohistoriques en verre pour la Péninsule armoricaine ${ }^{2}$.

En effet, ces bracelets ont rarement fait l'objet d'analyses poussées, aussi bien typochronologiques que chimiques. Aussi, cette double approche nous permet de dresser un premier bilan sur ce sujet en apportant des réponses aux multiples questions en suspens, telles que : les bracelets en verre sont-ils associables à un type de contexte? Quelle est leur datation? Existe t-il des liens entre typologie, datation et composition chimique? Où et comment ont-ils été produis? D'où proviennent les verres utilisés? Y a t-il eu des spécificités dans l'approvisionnement du verre en fonction des couleurs et/ou du temps?

Dans un premier temps, les bracelets inventoriés seront datés par des méthodes de comparaison et de typologie. Puis, dans un second temps, des analyses chimiques permettront de connaitre la composition de la pâte et les éléments chro-

*dinard4@gmail.com

**IRAMAT, UMR 5060, CNRS/Université d'Orléans, Centre Ernest-Babelon, 3D Rue de la Férollerie, 45071 Orléans cedex 2. (gratuze@cnrs-orleans.fr)

*** Inrap Grand-Ouest, UMR 6566, CNRS/Université de Rennes 1, 2 et Nantes/MCC, 37 rue du Bignon, 35577 Cesson-Sévigné. (anne-francoise.cherel@inrap.fr)

1. «Les premiers éléments de parure en faïence et en verre de la Bretagne " communication d'Anne-Françoise Cherel et de Bernard Gratuze lors du colloque de l'AFAV à Rennes les 30 novembre et $1^{\text {er }}$ décembre 2007 (résumés de communications, 2008, p. 8).

2. Le présent article est issu d'un mémoire de Master 2 préparé sous la direction de B. Gratuze et A.-F. Cherel (Dinard, 2009). 
mogènes propres à chaque bracelet. Ces mêmes analyses nous aiguilleront vers l'origine géographique probable de la matière première et apporteront d'autres données concernant l'approvisionnement en verre et les échanges liés à ce matériau.

\section{LE CORPUS}

Les bracelets en verre apparaissant vers le milieu du $\mathrm{III}^{\mathrm{e}}$ siècle avant notre ère sur le territoire armoricain, nous avons pris en considération la fin du second âge du Fer pour constituer le corpus. En datation absolue, la période s'étend de 300 à 30 ans avant notre ère.

Les recherches bibliographiques et les entrevues avec des archéologues professionnels et amateurs (majoritairement menées par Anne-Françoise Cherel) ont permis le recensement de 43 bracelets (ou fragments) en verre pour 24 sites archéologiques répartis sur l'ensemble du territoire breton (fig. 1 et tabl. 1). Leur répartition est très inégale : on note une concentration de ce type de mobilier en Morbihan (19 objets) et en Côtes-d'Armor (15). Les découvertes se répartissent entre trois principaux types de contextes : les habitats, les sépultures et les lieux de culte. En plus des fouilles, les prospections pédestres (ramassages hors d'une structure archéologique reconnue) ont également apporté du mobilier en verre.

\section{Méthodologie}

\section{Les typologies existantes et leurs limites}

Les bracelets inventoriés sont en verre bleu, vert, incolore avec une couche jaune, violet ou brunâtre (marron, brun et brun-rouge). Certains bracelets sont simples (avec ou sans moulures et bourgeons), d'autres sont ornés de filets ou zigzags de couleur bleue, blanche et/ou jaune. Tous ces paramètres associés rendent le nombre de possibilités et d'aspect très important; aussi, convient-il d'employer une typologie adéquate pour faciliter l'étude de ces mobiliers de parure.

Une approche typologique a été mise en place par Thea Elisabeth Haevernick (Haevernick, 1960), dont les travaux servent encore aujourd'hui de référence. Toutefois, cette méthode ne prenant en compte que les formes, Rupert Gebhard décida de la compléter en intégrant les critères d'ornementation, de couleurs et de proportions pouvant

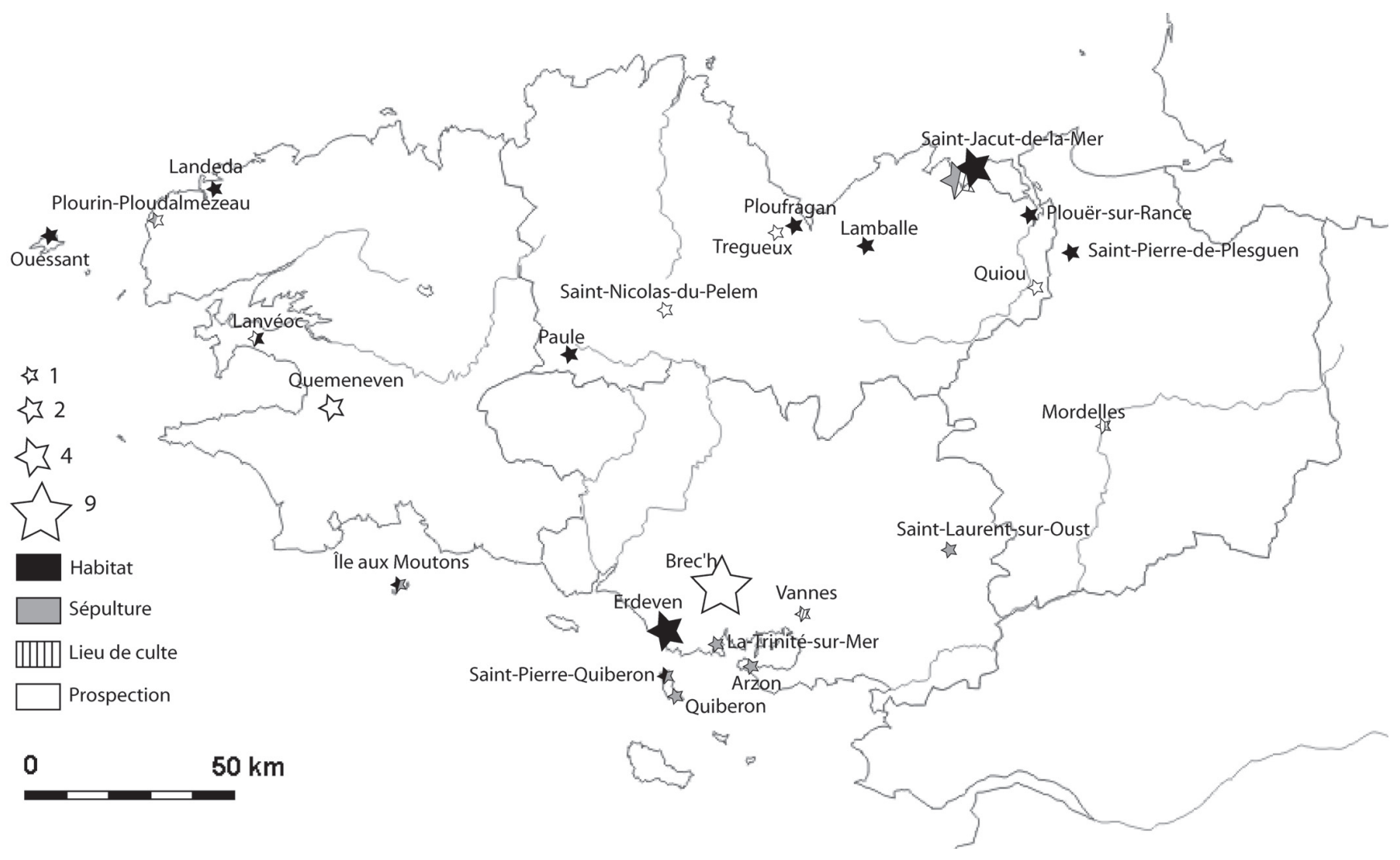

Figure 1 : Carte de répartition des bracelets protohistoriques en verre dans la péninsule bretonne.

Figure 1: Distribution map of protohistoric glass bracelets in the breton peninsula. 


\begin{tabular}{|c|c|c|c|c|}
\hline Département & $\begin{array}{c}\mathrm{Nb} \text {. de } \\
\text { bracelets }\end{array}$ & Site & Commune & Références \\
\hline \multirow{9}{*}{$\begin{array}{c}\text { Côtes } \\
\text { d'Armor } \\
(22)\end{array}$} & 1 & "Zac de La Tourelle» & Lamballe & Communication orale d'Anne-Françoise Cherel \\
\hline & 1 & & Le Quiou & Labaune et Petit-Aupert, 2005 \\
\hline & 1 & «Saint-Symphorien » & Paule & Menez, 2008 \\
\hline & 1 & "Le Boisanne" & Plouër-sur-Rance & Bizien-Jaglin et al., 2003; Menez, 1996 \\
\hline & 1 & "La rue des Bosses» & Ploufragan & Communication orale de Laure Simon \\
\hline & 4 & "Les Ebihens" & Saint-Jacut-de-la-Mer & Langouet et al., 1989; Bizien-Jaglin et al., 2003 \\
\hline & 4 & «Les Haches» & Saint-Jacut-de-la-Mer & $\begin{array}{l}\text { Bizien-Jaglin, 1992; Bizien-Jaglin et al., 2003; } \\
\text { Bizien-Jaglin et al., } 2004\end{array}$ \\
\hline & 1 & "Plateau du Collédic » & Saint-Nicolas-du-Pelem & Communication orale d'Anne-Françoise Cherel \\
\hline & 1 & "La Ville Pollo" & Trégueux & Dinard, 2008 \\
\hline \multirow{6}{*}{$\begin{array}{l}\text { Finistère } \\
\quad(29)\end{array}$} & 1 & "île Guennoc» & Landeda & Briard et al., 1979; Galliou, 1982 \\
\hline & 1 & "La Boissière " & Lanvéoc & Galliou, 1982 \\
\hline & 1 & "île aux moutons" & Fouessant & Communication orale de Marie-Yvane Daire \\
\hline & 1 & «Mez-Notariou » & Ouessant & Le Bihan, 2007 \\
\hline & 1 & "Kergadiou " & Plourin-Ploudalmézeau & Bousquet, 1961 ; Briard et al., 1979; Galliou, 1989 \\
\hline & 2 & «Kerfloux» & Quéménéven & Galliou et Pré, 2000; Le Goffic et Peuziat, 1997 \\
\hline \multirow{2}{*}{$\begin{array}{c}\text { Ille-et-Vilaine } \\
(35)\end{array}$} & 1 & "Sermon» & Mordelles & Batt, 1989 \\
\hline & 1 & «L'Homme Mort » & Saint-Pierre-de-Plesguen & Leroux et al., 1991 \\
\hline \multirow{8}{*}{$\begin{array}{l}\text { Morbihan } \\
\quad(56)\end{array}$} & 1 & "Le Petit Mont" & Arzon & Lecornec, 1994 \\
\hline & 9 & «La Lande du Rameau» & Brec'h & Clément et Galliou, 1985 \\
\hline & 4 & "Kerhilio " & Erdeven & Jacq, 1941 \\
\hline & 1 & "Mané-Roullarde " & La Trinité-sur-Mer & Clément et Galliou, 1985; Miln, 1882 \\
\hline & 1 & « Kerné» & Quiberon & Jacq, 1941 \\
\hline & 1 & «Beaumont» & Saint-Laurent-sur-Oust & Tinevez et al., 1990 \\
\hline & 1 & « Kergroix & Saint-Pierre-de-Quiberon & Galliou, 1982; Sanquer, 1975 \\
\hline & 1 & "Bilaire " & Vannes & Simon, 2001 \\
\hline
\end{tabular}

Tableau 1 : Corpus des bracelets protohistoriques en verre de la région Bretagne pris en compte dans l'étude.

Table 1: Corpus of the protohistoric glass bracelets studied from the Breton peninsula.

présenter une valeur chronologique (Gebhard, 1989a). Nous avons tout d'abord tenté d'associer ces deux typologies afin de classer l'ensemble des bracelets bretons.

Quelques bracelets ne possédant pas d'iconographie et/ou n'étant pas disponibles à la consultation (lieux de conservation incertains ou inaccessibles), ils n'ont pas pu être intégré à cette typologie; à savoir celui de "Kerfloux " (bracelet 2) dans le Finistère et ceux de "Kerné " et de "Kergroix " (Sanquer, 1975, p. 346-347) dans le Morbihan. Les autres ont pu être regroupés au sein d'un tableau (tabl. 2). Celui-ci a révélé des individus n'entrant dans aucune des deux typologies précitées; il s'agit de ceux provenant des sites de "Kerhilio " (bracelets 1, 2 et 3), de « La Boissière " et de "Kerfloux » (bracelet 1), regroupés en fin de tableau. Une première approche comparative n'a pas permis l'établissement d'analogies entre ces bracelets et d'autres existant en Europe. Ces cinq objets posent donc des difficultés puisqu'aucune association typologique n'a pu être faite les concernant. Afin d'étudier tous les bracelets armoricains, il convenait donc de mettre en place une nouvelle typologie.

La typologie d'Haevernick ne prend en compte que la section ou coupe, ce qui n'est pas suffisant. Quant à celle de Gebhard, si elle tient compte d'un ensemble élargi de paramètres élémentaires (section, couleur et décor), il reste difficile de la compléter; en effet l'auteur suggérait, à chaque découverte d'un bracelet non présent dans sa typologie (établie à partir des bracelets de Manching en Bavière), d'ajouter de nouveaux numéros à la suite des 95 formes répertoriées (Gebhard, 1989a, p. 77). En pratique, cela demeure plus compliqué qu'il n'y parait puisque, par manque de communication entre chercheurs, un même type de bracelet peut recevoir deux numéros distincts et, inversement, on peut attribuer le même numéro à deux bracelets différents. De plus, on peut rapidement se tromper en confondant « type 


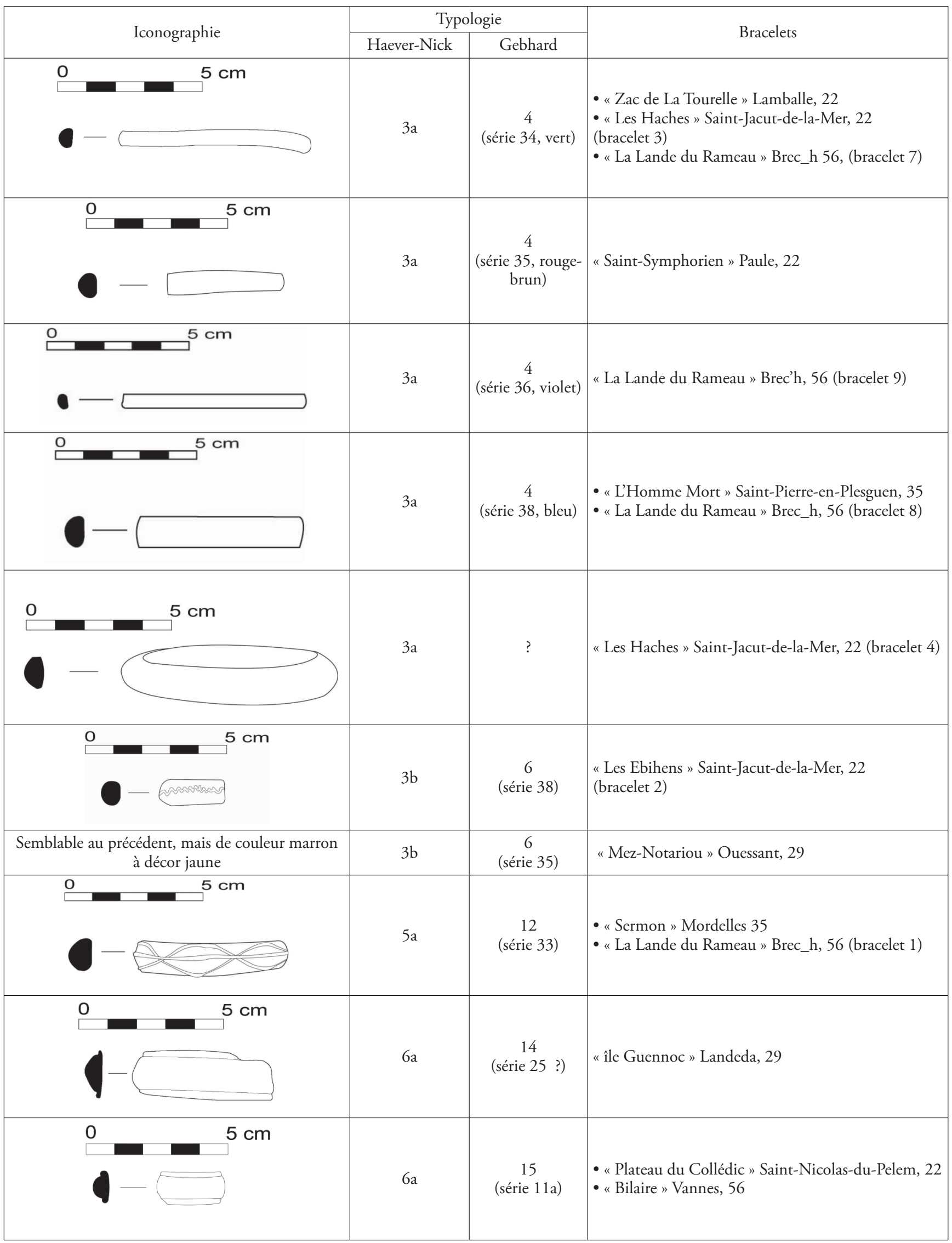




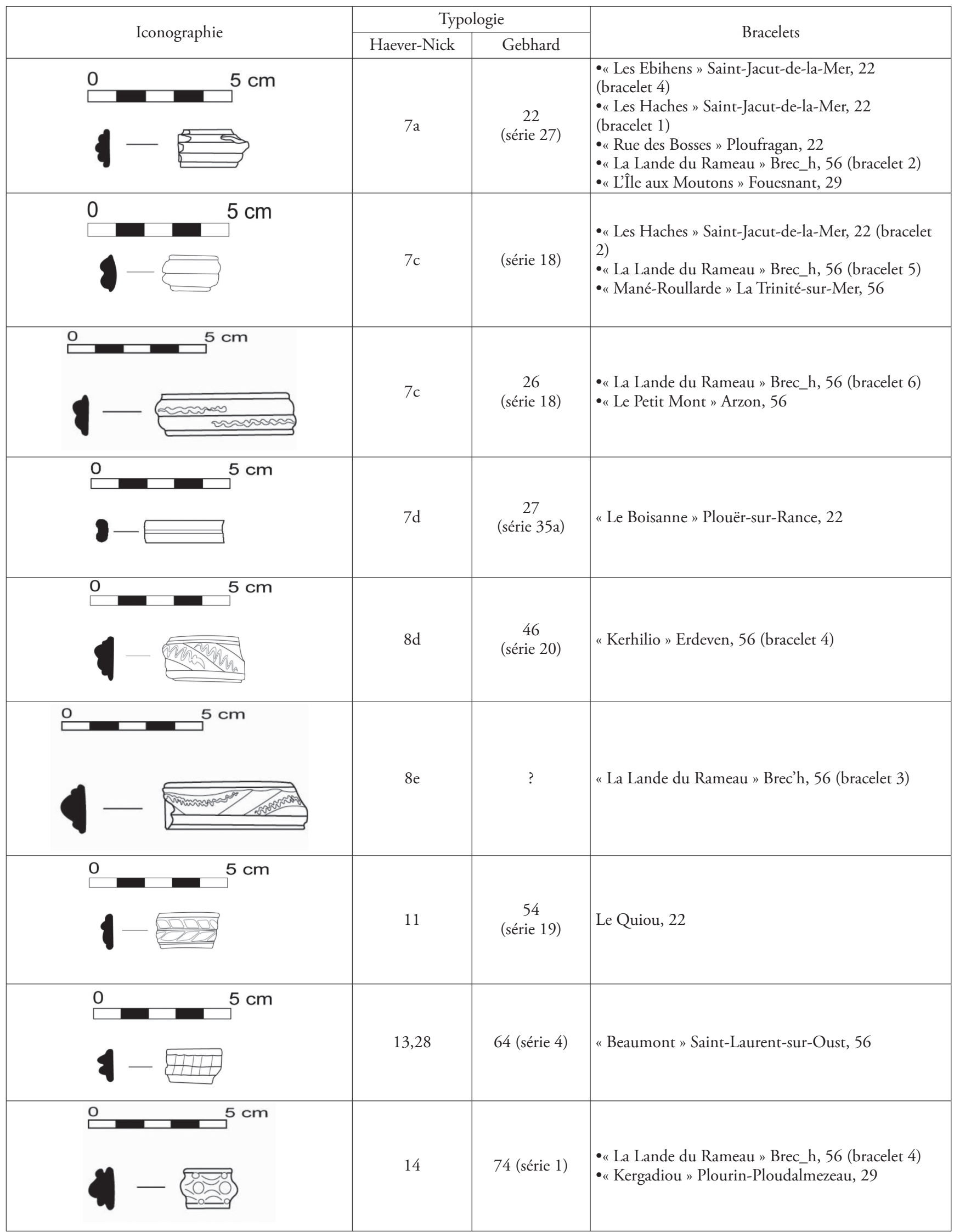




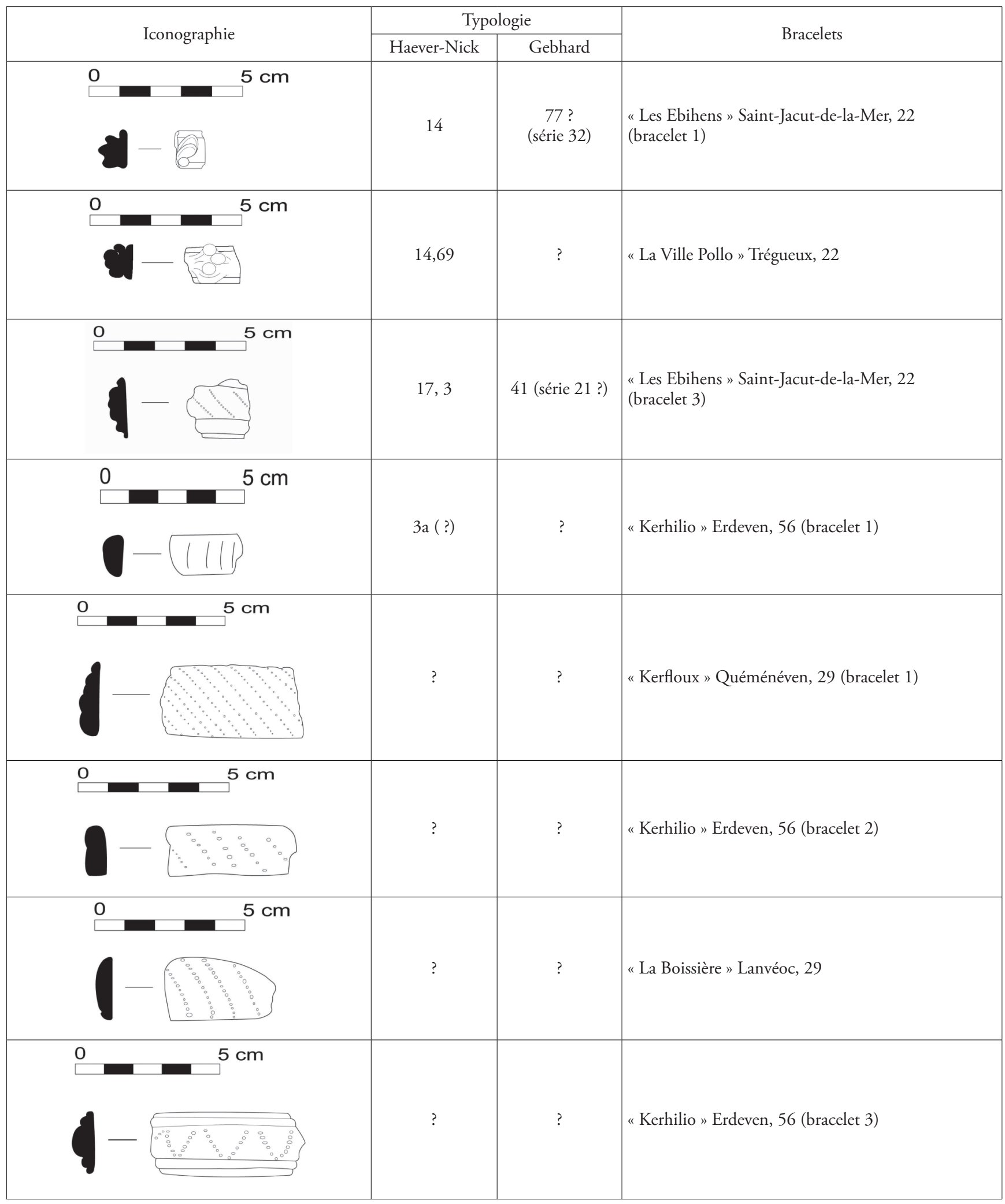

Tableau 2 : Inventaire des bracelets étudiés et correspondance avec les typologies de T. E. Haevernick (1960) et R. Gebhard (1989). Table 2: Inventory of the studied bracelets and relationship to the typologies of T. E. Haevernick (1960) and R. Gebhard (1989). 
Gebhard " (défini sur l'une des 95 formes décrites) et « série Gebhard " (issue du regroupement par l'auteur de quelques «types» au sein d'une série).

La solution proposée ici serait de mettre en place une sorte de code traduisant chaque bracelet en une formule qui tiendrait compte de la section, de la couleur et du décor. Ainsi, l'idée de Gebhard serait respectée, puisqu'on pourrait associer une chronologie à cette formule typologique. L'idéal est donc d'établir un code qui puisse s'appliquer pour tous les bracelets de tous les sites, en Bretagne et ailleurs.

\section{Codification typologique}

Les bracelets ont été décrits depuis les cordons latéraux vers le cordon central. Les " codes" s'organisent en trois temps : d'abord la coupe ou section, puis la couleur principale et enfin les décors (forme et couleur). On aura ainsi une idée du bracelet, d'abord générale (la coupe), puis de plus en plus précise (couleur et décors). Certains groupes d'Haevernick ou de Gebhard mentionnés ici ne sont pas représentés pas dans le corpus étudié. Cela montre que chaque bracelet, breton ou non, peut être codifié dans le système proposé (tabl. 3) et permet, à partir des anciennes typologies, de préciser les correspondances avec la nouvelle codification.

La section est définie par une ou plusieurs lettres majuscules, choisies selon l'aspect visuel du cordon ou bracelet :

$-\mathrm{D}$ pour les bracelets à profil simple en « $\mathrm{D}$ » (ex : groupes Haevernick 1 à 3).

- C pour les cordons simples et longitudinaux (tels que les groupes 6 et 7 d'Haevernick).

- T pour les cordons torsadés (tel que le cordon central du groupe 8 d'Haevernick).

- I pour les cordons divisés verticalement (tel que le groupe 9 d'Haevernick ou les types 49 et 67 de Gebhard).

$-\mathrm{V}$ pour les cordons figurant des chevrons (tels que les bracelets 31 et 48 de Gebhard).

- $S$ pour les cordons serpentiformes (généralement des moulures, tels que les types 80, 86 et 92 de Gebhard).

- W pour les bracelets figurants des vagues (tel que le groupe 4 d'Haevernick).

- B pour les cordons possédant des bourgeonnements, des boules (tels que les groupes 12 et 14 d'Haevernick).

- L pour les cordons marqués par des losanges (tel que le type 79 de Gebhard).

- E pour les cordons à l'aspect d'entrelacs ou de forme libre (tels que les types 83 et 88 de Gebhard).

Les couleurs sont traduites par des lettres minuscules:

- b bleu (blue)

- w blanc (white)

- bo brun, marron, ambre (brown)

- p pourpre, violet (purple) $-r$ rouge (red)

- g vert (green)

$-\mathrm{y}$ jaune (yellow)

- $\mathrm{o}$ orange

- in incolore

- iny bracelet incolore dont la face intérieure est revêtue

d'une fine couche de verre jaune opaque.

Les décors sont illustrés par d'autres lettres minuscules :

- $\mathrm{f}$ pour les décors en forme de filet ou de filament (par exemple, les filets bleus rajoutés du groupe 5 d'Haevernick).

- z pour les décors en forme de zigzag (filets beaucoup plus resserrés et anguleux que les filaments " $f$ »); ils sont généralement, là aussi, rajoutés.

- oc pour les décors en forme d'ocelle.

-1 pour les décors de forme libre.

Aux lettres, on ajoutera des chiffres (placés devant ces lettres) pour déterminer le nombre de cordons. Ainsi que deux signes : / (le slache), signifiant «à la fois " et * (l'étoile), pour traduire l'alternance. L'emploi du point sert à séparer ces trois groupes de paramètres.

Pour favoriser la compréhension de ce système, voici deux exemples :

- Tout d'abord, le bracelet bleu du « Petit Mont » à Arzon, qui possède quatre cordons dits simples et des décors de zigzag jaune et blanc. En mode codé, cela nous donne 4C. b. zy* ${ }^{*}$ w, soit $4 \mathrm{C}$ pour 4 cordons, b pour la couleur bleue et $\mathrm{zy}^{*} \mathrm{zw}$ signifiant qu'il y a à la fois des zigzags jaunes et des zigzags blancs (généralement, ceux-ci s’alternent).

- Pour le bracelet bleu bourgeonnant de Trégueux, on aurait le code $2 \mathrm{C} 3 \mathrm{~B}$. b, c'est à dire $2 \mathrm{C}$ pour les 2 cordons latéraux et $3 \mathrm{~B}$ pour la séquence (répétitive) de 3 bourgeons implantés sur le corps du bracelet, puis b pour la couleur.

Lensemble des bracelets de notre corpus qui ont pu être examinés (cf. supra) a été retranscrit de cette façon (tabl. 4).

\section{Comparaisons typo-chronologiques}

\section{Les formes simples à section en $D$}

Plusieurs bracelets sont regroupés ici. Leur section est simple et leur taille varie.

Les bracelets bleus de ce groupe (tabl. 4, I) sont ceux des "Haches » ( $\left.n^{\circ} 4\right)$ à Saint-Jacut-de-la-Mer (Côtes-d'Armor), de "L'Homme Mort " à Saint-Pierre-de-Plesguen (Illeet-Vilaine) et de "La Lande du Rameau " ( $\left.n^{\circ} 8\right)$ à Brec'h (Morbihan). Le bracelet de L'Homme Mort (Leroux et al., 1991) a été mis au jour à l'emplacement des vestiges d'un bâtiment gaulois. Celui des Haches était porté par une femme inhumée au sein d'une petite nécropole située sur une petite butte de terre et celui de La Lande du Rameau appartient à un dépôt découvert fortuitement. Les auteurs ont daté les bracelets des Haches et de L'Homme Mort par 


\begin{tabular}{|c|c|c|}
\hline $\begin{array}{l}\text { Typologie } \\
\text { Haervernick }\end{array}$ & Typologie Gebhard & Code \\
\hline 1,2 et $3 a$ & $\begin{array}{c}1 \text { à } 5 \\
(\text { série } 34,35,36,37,38 \text { et 39) }\end{array}$ & D. (lettre couleur) \\
\hline $3 b$ & 6 & D. (lettre couleur). z (couleur) \\
\hline $3 c$ & 7 & D. (lettre couleur). oc (couleur) \\
\hline $3 \mathrm{~d}$ & 8 & D. (lettre couleur)/(lettre couleur) \\
\hline 4 & 9 & W. (lettre couleur)/(lettre couleur) \\
\hline $5 \mathrm{a}$ & 10 (série 33 naturel) & D. (lettre couleur). (nombre)f (couleur). \\
\hline $5 b$ & 12 & 3C. (lettre couleur). (nombre)f (couleur) \\
\hline $6 a$ et $6 c$ & 13 à 15 et 18 (série 25,26 et $11 \mathrm{~B}$ ) & 3C. (lettre couleur) \\
\hline $6 \mathrm{~b}$ & 16 (et 17 ?) (série 11Aet 11B) & 3C. (lettre couleur). z (couleur) \\
\hline $6 b$ & & $\begin{array}{l}\text { 3C. (lettre couleur). } 1 \text { (couleur) } \\
\text { Ex : Manching (Allemagne) }\end{array}$ \\
\hline $7 \mathrm{a}$ & 19 à 22 (série 27 et 17 ) & 5C. (lettre couleur) \\
\hline $7 \mathrm{~b}$ & 23 à 25 (série 15 et 14 ) & 5C. (lettre couleur). z (couleur) \\
\hline $7 \mathrm{c}$ & 26 (série 18 b) & $\begin{array}{l}\text { 4C. (lettre couleur). z (couleur) } \\
\text { et 4C. (lettre couleur) }\end{array}$ \\
\hline $7 \mathrm{~d}$ & 27 (série 34A, 35A, 36A et 38A) & 2C. (lettre couleur) \\
\hline $8 \mathrm{a}$ & 28 à 30 & 2CT. (lettre couleur) \\
\hline $8 \mathrm{a}$ & 31 & 2CV. (lettre couleur) \\
\hline $8 \mathrm{~b}$ & 32 à 37 (série 12 et 13 ) & 2CT. (lettre couleur). z (couleur) \\
\hline $8 \mathrm{~b}$ & 38 & $\begin{array}{l}\text { 2CT. (lettre couleur). z (couleur)* } \\
\text { oc (couleur) }\end{array}$ \\
\hline $8 c, 10$ et 17,3 & 39 à 44 et 53 (série 21$)$ & $\begin{array}{c}\text { 4CT. (lettre couleur) } \\
\text { Ex : Saint Jacut de la Mer (22), 4CT. b. o } \\
\text { Ex : Besançon (25) }\end{array}$ \\
\hline $8 \mathrm{~d}$ & 45 à 47 (série 20) & 4CT. (lettre couleur). z (couleur) \\
\hline $8 \mathrm{e}$ & 48 & 4CV/T. (lettre couleur). z (couleur) \\
\hline \multirow[t]{3}{*}{17} & & $\begin{array}{l}\text { 4CV/T. (lettre couleur) } \\
\text { Ex : Mandeure }(25)\end{array}$ \\
\hline & & $\begin{array}{l}\text { 4CV. (lettre couleur) } \\
\text { ex : Erdeven }(56)\end{array}$ \\
\hline & & $\begin{array}{l}\text { 5C/V. (lettre couleur) } \\
\text { Ex : Besançon }(25)\end{array}$ \\
\hline 9 et 13 & 49 et $61(\mathrm{H} 9=$ série 10 et 28$)$ et $(\mathrm{H} 13=$ série 9$)$ & 2CI. (lettre couleur) \\
\hline 10 & 50 (série 30) & 3C2I. (lettre couleur) \\
\hline 10 & 51 et 85 (série 22 et 31 ) & 2C2IT. (lettre couleur) \\
\hline 10 et 13 & 52 et 71 & 2C3T. (lettre couleur) \\
\hline 11 et 13 & 54 et 68 (H11=série 29$)$ & 2C2T. (lettre couleur) \\
\hline 11 & 55 (série 19) & 2C2T. (lettre couleur). z (couleur) \\
\hline 12 & 56 et 59 & 2CB. (lettre couleur) \\
\hline 12 & 57 (série 3) et 58 (série 6) & B. (lettre couleur) \\
\hline 12 & 60 & 2C2IB. (lettre couleur) \\
\hline 13 & 62 & 4CI. (lettre couleur) \\
\hline 13 & 63 & 4CI. (lettre couleur). z (couleur) \\
\hline 13 & 64 et 67 (série 4) & 2C2I. (lettre couleur) \\
\hline 13 & 65 et 66 & 4C2I. (lettre couleur) \\
\hline 13 & 69 & 4C2T. (lettre couleur) \\
\hline
\end{tabular}




\begin{tabular}{|c|c|c|}
\hline $\begin{array}{c}\text { Typologie } \\
\text { Haervernick }\end{array}$ & Typologie Gebhard & Code \\
\hline 13 & 70 et 87 & 4C2B. (lettre couleur) \\
\hline 14 & 72 & 4B. (lettre couleur) \\
\hline 14 & 73 (série 1 ) & 2B. (lettre couleur) \\
\hline 14 & 74 à 76 & 3B. (lettre couleur) \\
\hline 14 & 77 et 82 (série 3) & 2C2B. (lettre couleur) \\
\hline 14 & & $\begin{array}{c}\text { 2C3B. (lettre couleur) } \\
\text { Ex : Nages (30), Saint-Jacut-de-la-Mer (22) }\end{array}$ \\
\hline 14 & & $\begin{array}{c}\text { 2C4B. (lettre couleur) } \\
\text { Ex : Nages (30), Trégueux (22), } \\
\text { Epiais-Rhus (95) }\end{array}$ \\
\hline 14 & 78 (série 24 et série 32 ) & 4C2B/T. (lettre couleur) \\
\hline 15 & 79 & 2CL/B*2B. (lettre couleur). z (couleur) \\
\hline 16 & 80 (série 23) & 4CS. (lettre couleur) \\
\hline \multirow[t]{15}{*}{17} & 84 & 4CI*C. (lettre couleur) \\
\hline & 81 & 2C3BT. (lettre couleur) \\
\hline & 83 & 2CE. (lettre couleur) \\
\hline & 86 & $\begin{array}{l}\text { 4CI*S ou 4CS/I. (lettre couleur) } \\
\text { [selon les bracelets] }\end{array}$ \\
\hline & 88 & 4CE. (lettre couleur) \\
\hline & 89 & 3C2B. (lettre couleur) \\
\hline & 90 & 4CB. (lettre couleur) \\
\hline & 91 & 3CIT. (lettre couleur) \\
\hline & 92 & 2CS. (lettre couleur) \\
\hline & 93 & 2CI*T. (lettre couleur) \\
\hline & 94 & $\begin{array}{l}\text { D/S. (lettre couleur) ou D. } \\
\text { (lettre couleur). (nombre) f (couleur) } \\
\text { [selon les bracelets]. }\end{array}$ \\
\hline & 95 & 2BT. (lettre couleur) \\
\hline & & $\begin{array}{l}\text { I. (lettre couleur) } \\
\text { Ex : Erdeven (56) }\end{array}$ \\
\hline & & $\begin{array}{c}\text { T. (lettre couleur) } \\
\text { Ex : Erdeven (56) et Lanvéoc (29) }\end{array}$ \\
\hline & & 3I. (lettre couleur) Ex : Nages (30) \\
\hline
\end{tabular}

Tableau 3 : Proposition de codage typologique des bracelets en verre étudiés.

Table 3: Proposed typological scheme of the glass bracelets studied.

comparaisons; le dépôt de Brec'h a été daté d'après du mobilier monétaire marquant la transition La Tène D2/période gallo-romaine (Clément et Galliou, 1985). Des bracelets bleus de même section sont datés de La Tène $\mathrm{D}$ sur le site de Nage dans le Gard (Feugère et Py, 1989).

Les bracelets verts du type D (tabl. 4, II) sont ceux de la

"Zac de la Tourelle " à Lamballe (Côtes-d'Armor), des "Haches" ( $\left.n^{\circ} 3\right)$ et de "La Lande du Rameau " ( $\left.n^{\circ} 7\right)$. Celui de la Tourelle est issu d'un contexte cultuel de La Tène $\mathrm{C}$ (communication Anne-Françoise Cherel). Le n ${ }^{\circ} 3$ des Haches provient d'un comblement remanié sur le lieu de culte, ce qui ne permet pas de lui attribuer une datation plus précise que la fin du second âge du Fer (Bizien-Jaglin, 1992; 2004). Comme indiqué plus haut, le dépôt de la Lande du Rameau est attribué à la transition La TèneD2/ Gallo-romain.

Un bracelet marron-ambré à section en $\mathrm{D}$ provient de "Saint-Symphorien » à Paule dans les Côtes-d'Armor (tabl. 4, III). Il a été mis au jour dans les décombres de l'incendie d'une forteresse et est associé à la phase 4 du site (Menez, 2008), datée de La Tène C2. Cependant, à EpiaisRhus (Val-d'Oise), les bracelets de cette couleur sont attri- 


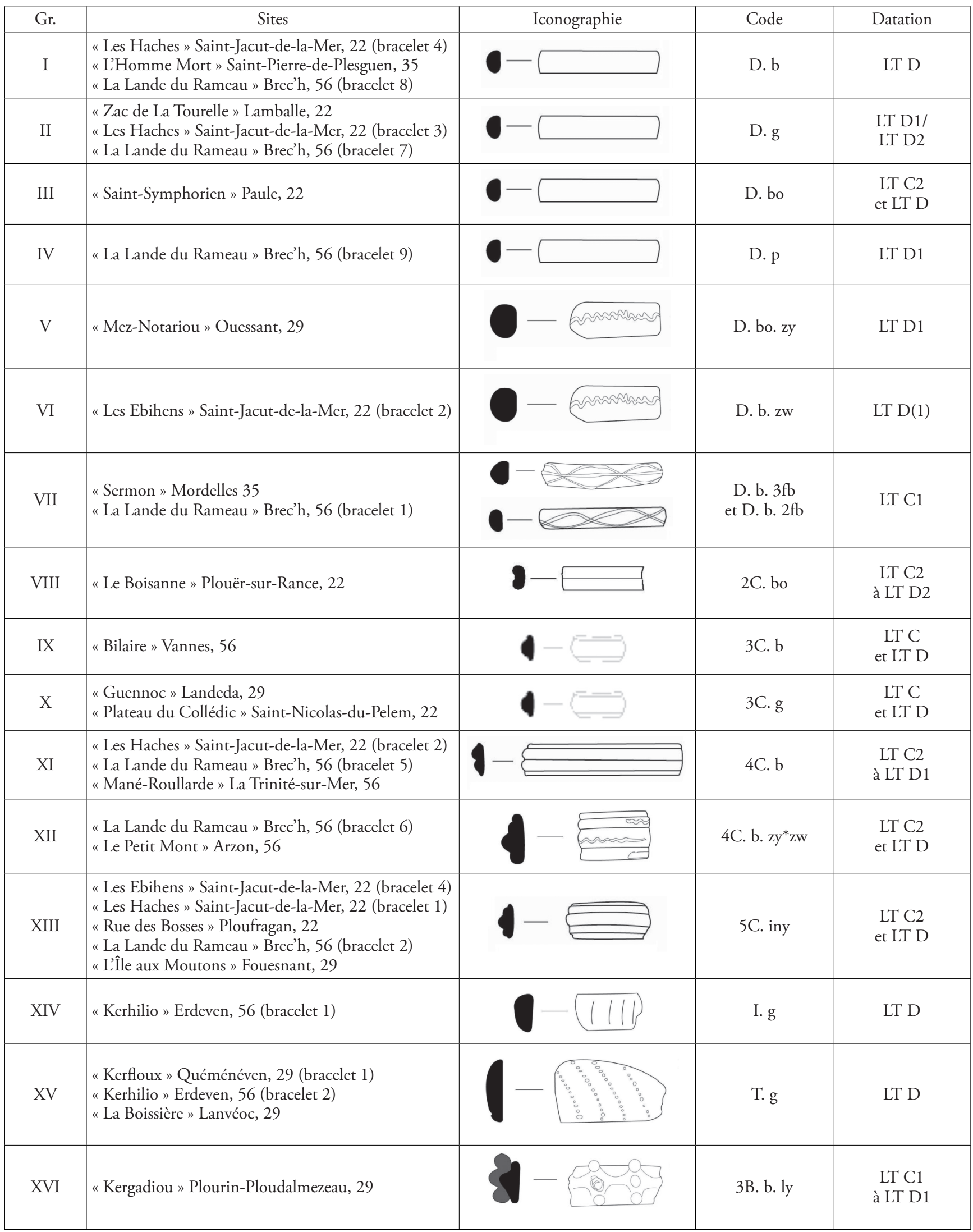




\begin{tabular}{|c|c|c|c|c|}
\hline Gr. & Sites & Iconographie & Code & Datation \\
\hline XVII & Le Quiou, 22 & - & 2C2T. b & $\begin{array}{l}\text { LT C1 } \\
\text { à LT D1 }\end{array}$ \\
\hline XVIII & «Beaumont » Saint-Laurent-sur-Oust, 56 & & 2C2I. b & $\begin{array}{c}\text { LT C } \\
\text { et LT D }\end{array}$ \\
\hline XIX & $\begin{array}{l}\text { "La Lande du Rameau » Brec’h, } 56 \text { (bracelet 4) } \\
\text { «Les Ebihens » Saint-Jacut-de-la-Mer, } 22 \text { (bracelet 1) }\end{array}$ & & 2C3B. b & $\begin{array}{l}\text { LT C2 } \\
\text { et LT D }\end{array}$ \\
\hline $\mathrm{XX}$ & « La Ville Pollo » Trégueux, 22 & & 2C4B. b & LT D \\
\hline XXI & «Les Ebihens » Saint-Jacut-de-la-Mer, 22 (bracelet 3) & & 4CT. b & $\begin{array}{l}\text { LT C2 } \\
\text { à LT D2 }\end{array}$ \\
\hline XXII & « Kerhilio » Erdeven, 56 (bracelet 4) & & 4CT. b. zy*zw & $\begin{array}{c}\text { LT C } \\
\text { et LT D }\end{array}$ \\
\hline XXIII & « Kerhilio » Erdeven, 56 (bracelet 3) & & $4 \mathrm{CV} . \mathrm{b}$ & LT D \\
\hline XIV & «La Lande du Rameau » Brec’h, 56 (bracelet 3) & & 4CV/T. b. $\mathrm{zx}^{*} \mathrm{zy}$ & LT D1 \\
\hline
\end{tabular}

Tableau 4 : Dessin, codage typologique et datation des bracelets en verre bretons.

Table 4: Drawing, typological scheme and dating of Breton glass bracelets.

bués à La Tène D (Vanpeene, 1989). Notons enfin que de tels bracelets, bleus, pourpres et marron-ambré, sont connus à La Tène D1 à Bibracte (Saône-et-Loire) (Bride, 2005) et, à La Tène D, à « Mandeure " (Doubs) (Guillard, 1989).

Le bracelet pourpre de notre série est le $\mathrm{n}^{\circ} 9$ de La Lande du Rameau (tabl. 4, IV). Des bracelets à section en D de couleur pourpre sont connus sur les sites de Nage (Feugère et Py, 1989) et du " Village des Arènes " à Levroux (Indre) où ils sont associés à La Tène $\mathrm{D}$ (Tilliard, 1989). Ces bracelets à section en $\mathrm{D}$, bleus et pourpres, trouvent aussi des références à La Tène D à Manching en Allemagne (Gebhard, 1989b). Cette datation est renforcée par l'apparition de la couleur pourpre dans le verre, qui se fait à cette période (Venclovà, 1989).

\section{Les bracelets à section en $D$ ornés}

a) bracelets à zigzag rapporté jaune ou blanc (D.b.zw et D.bo.zy)

Les bracelets correspondant à ces types (tabl. 4, V et VI) sont ceux de " Mez-Notariou » à Ouessant (Finistère) et des "Ebihens » (n 2) à Saint-Jacut-de-la-Mer (Côtes-d'Armor). Le premier - D.bo.zy - a été mis au jour dans l'Us 311 de l'habitat du Bronze moyen (Le Bihan, 2007). Cette unité stratigraphique contenait également des tessons des premier et second âges du Fer, deux fragments de verre romain et deux tessons modernes. L'auteur l'a daté de La Tène D1 par comparaison avec des bracelets d'autres sites et en fonction du mobilier associé. Le second bracelet - D.b.zw - provient de l'habitat, matérialisé par des murets et situé sur un petit éperon rocheux, des Ebihens (Langouet et al., 1989).

Sur le site de Nage (Gard), les bracelets à section en D décorés de zigzags sont associés à La Tène D1b (Feugère 
et Py, 1989).Des bracelets comparables (bleus, pourpres et brun-ambrés) présentant le même décor sont attribués à $\mathrm{La}$ Tène D sur le site de Mandeure (Doubs) (Guillard, 1989). De couleur pourpre, ils sont connus à La Tène $\mathrm{D} 1$ à Bibracte (Saône-et-Loire) (Bride, 2005).

b) bracelets bleu clair à filets rajoutés de couleur bleu foncé (D.b.3fb et D.b.2fb).

L'exemplaire orné de trois filets a été trouvé sur l'occupation gauloise du temple gallo-romain de Sermon à Mordelles (Ille-et-Vilaine) (Batt, 1987). L'auteur semble avoir daté ce bracelet par l'emploi de comparaisons. Celui qui est orné de deux filets est le $\mathrm{n}^{\circ} 1$ du dépôt de La Lande du Rameau à Brec'h (Clément et Galliou, 1985) (tabl. 4, VII).

Les bracelets illustrant ce type trouvent des comparaisons avec des parures exhumées dans le canton de Fribourg en Suisse (Ramseyer, 1995), à Manching (Gebhard, 1989b) et sur le site de "La Vache à l'Aise » à Bobigny en Seine-SaintDenis (Le Béchennec et al., 2007); ils sont datés de La Tène C1. Il est établi que ce type figure parmi les plus anciens, avec une attribution à La Tène C1 (Kaenel et Müller, 1989); il semble pourtant perdurer jusqu'à la fin de La Tène D (Batt, 1987).

\section{Les bracelets à cordons multiples}

a) Bracelets à double cordon (2C.bo).

De couleur ambre, notre représentant de ce type (tabl. 4, VIII) a été mis au jour dans un fossé du "Boisanne " à Plouër-sur-Rance (Côtes-d'Armor) (Menez, 1996; Bizien et al., 2003). Il se trouvait dans une structure datée de la transition La Tène C2/La Tène D1.

Sans indication de couleur, le bracelet à double cordon trouve des références de La Tène C2 à la fin de La Tène D2 sur le site de Mandeure (Doubs) (Guillard, 1989). De couleur verte, le fragment à double cordon provenant du sanctuaire de "Mars Mullo " à Allonnes (Sarthe) est attribué à La Tène $\mathrm{C}$ (Brouquier-Reddé et Gruel, 2004). De couleur brune, ce type existe à La Tène $\mathrm{D}$ à Manching en Allemagne (Gebhard, 1989b). Pourpre, il est connu à La Tène C2 à Nage dans le Gard (Feugère et Py, 1989).

\section{b) Bracelets à triple cordon.}

Les trois bracelets bretons de ce groupe répondent aux formules 3C.b et 3C.g. (tabl. 4, IX et X). Dans sa version bleue, ce type a été mis au jour sur une occupation gauloise à proximité du sanctuaire gallo-romain du "Bilaire » à Vannes (Morbihan) (Simon, 2001). Sur le site de Nage, ce type est associé à un contexte de La Tène C2 jusqu’à la fin de La Tène D1 (Feugère et Py, 1989).

De couleur verte, deux d'entre eux ont été trouvés sur les sites de "l'île Guennoc " à Landeda (Finistère) (Galliou,
1982) et du "Plateau du Collédic " à Saint-Nicolas-duPélem (Côtes-d'Armor). Dans les deux cas, nous manquons d'informations au sujet du contexte de découverte.

\section{c) Bracelets à quadruple cordon.}

Les fragments de bracelets bleus à cordons simples (4C.b) sont ceux des "Haches " ( $\left.n^{\circ} 2\right)$ à Saint-Jacut-de-la-Mer (Côtes-d'Armor), de «La Lande du Rameau " (n $\left.{ }^{\circ} 5\right)$ à Brec'h et de "Mané-Roullarde » en La Trinité-sur-Mer, deux sites du Morbihan (tabl. 4, XI). Le bracelet des Haches provient d'un contexte remanié sur un lieu de culte (Bizien-Jaglin, 1992 et 2004). Les bracelets morbihannais ont été trouvés lors de prospections, celui de Mané-Roullarde ayant été découvert près d'un tertre (Miln, 1882).

Pour une datation du début La Tène C2 à la fin de La Tène D1, des parallèles sont possibles avec les bracelets du "Pâtural " à côté de Clermont-Ferrand (Puy-de-Dôme) (Robinson, 2003).

Les bracelets bleu cobalt avec quatre cordons (les cordons centraux étant généralement plus épais que ceux les encadrant) et zigzags (blancs et/ou jaunes) incrustés sur certains de ces cordons (type 4C.b.zy ${ }^{*} \mathrm{zw}$ ) sont illustrés par deux bracelets morbihannais (tabl. 4, XII). Il s'agit de ceux de " La Lande du Rameau " ( $n^{\circ}$ 6) à Brec'h et du "Petit Mont " à Arzon; ce dernier a été recueilli au sein d'un milieu perturbé en avant du portique d'entrée du dolmen IIIA (Lecornec, 1994, p. 68-69 \& pl. h.-t.).

Ces bracelets trouvent des comparaisons à La Tène C2, sur les sites de La Vache à l'Aise à Bobigny (Seine-Saint-Denis) (Le Béchennec et al., 2005), de La Villeneuve-au-Châtelot (Aube) (Bataille, 2008), du Village des Arènes à Levroux (Indre) (Tilliard, 1989) et de Nage (Gard) (Feugère et Py, 1989). A Epiais-Rhus (Val-d'Oise), un bracelet de ce type est associé à La Tène D (Vanpeene, 1989).

\section{d) Bracelets à cinq cordons (type 5C.iny).}

Les exemplaires bretons sont constitués de trois cordons centraux encadrés par deux fins cordons latéraux; leur face intérieure est revêtue d'une fine couche de verre jaune opaque.

Ce type est le plus représenté dans la Péninsule armoricaine (tabl. 4, XIII). Ce sont le bracelet morbihannais de "La Lande du Rameau " (n²) à Brec'h (dépôt), l'exemplaire finistérien de "l'Île aux Moutons » à Fouesnant et les parures costarmoricaines de "La Rue des Bosses " à Ploufragan (habitat), des "Ebihens " $\left(n^{\circ} 4\right)$ et des " Haches " $\left(n^{\circ} 1\right)$ à Saint-Jacut-de-la-Mer. Ce dernier provient d'un contexte remanié sur un lieu de culte (Bizien-Jaglin, 1992; 2004). Le bracelet de l'Île aux Moutons se trouve associé à un contexte de La Tène D, mêlant habitat et espace funéraire. Les quatre autres ont été datés par comparaison avec ceux d'autres sites. 
Sur le site de "Mars Mullo » à Allonnes (Sarthe), le type 5C.iny est connu à La Tène C (Brouquier-Reddé et Gruel, 2004). Il est attribué à La Tène $C 2$ sur les sites de Nage (Gard) (Feugère et Py, 1989) et de Mandeure (Doubs) (Guillard, 1989). Un bracelet présentant les mêmes couleurs est daté entre La Tène $\mathrm{C} 2$ et à La Tène D1 sur le site du Pâtural près de Clermont-Ferrand (Puy-de-Dôme) (Robinson, 2003).

\section{Formes incisées, torsadées ou bourgeonnantes}

- Type I.g (tabl. 4, XIV). Le bracelet vert de Kerhilio ( $\left.{ }^{\circ} 1\right)$ est un cordon sur lequel on a imprimé des coups transversaux peu profonds. Il est daté de La Tène D sur ce site d'habitation d'Erdeven (Morbihan), mais sans précision sur le contexte de découverte dans l'ouvrage de M. Jacq (1941, p. 167).

- Type T.g (tabl. 4, XV). Ces bracelets verts, à unique cordon torsadé, sont ceux de "Kerhilio " ( $\left.\mathrm{n}^{\circ} 2\right)$ à Erdeven (Morbihan), de «Kerfloux » ( $\left.n^{\circ} 1\right)$ à Quéménéven et de « La Boissière " à Lanvéoc (deux sites finistériens). Le bracelet de Kerfloux (Pré et Galliou, 2000, p. 40 ; Le Goffic et Peuziat, 1997, p. 59) a été trouvé lors de prospections, ainsi que celui de La Boissière (Galliou, 1982 et 1989). Ce type a été attribué à La Tène III, ce qui équivaut à La Tène D sur le site Kerhilio (Jacq, 1941).

- Type 3B.b.ly (tabl. 4, XVI). L'unique exemplaire de ce type est orné de séquences à trois bourgeons, dont l'un semble orné d'un décor libre de teinte jaune. Il s'agit de l'objet recueilli lors de prospections aux environs immédiats du grand menhir de Kergadiou à Plourin-Ploudalmezau (Finistère) (Bousquet, 1961, p. 351; Giot et al., 1995, p. 303; Galliou, 1989). Un autre bracelet bleu, dont la séquence est constituée de trois bourgeons, est daté de La Tène C1 à la fin de La Tène D1 sur le site de Nage (Gard) (Feugère et Py, 1989).

- Type 2C2T.b (tabl. 4, XVII). Ce bracelet bleu est constitué de deux cordons torsadés encadrés par deux cordons simples. Il a été découvert lors de prospections aux alentours de la villa gallo-romaine du Quiou dans les Côtes-d'Armor (Labaune et Petit-Aupert, 2005).

Sur les sites de Pra Perrey à Gumefens (Fribourg, Suisse) et de Manching en Bavière, ce type est daté de La Tène C1 (Schwab, 1995; Gebhard, 1989b). Les bracelets bleus de type 2C2T de l'oppidum de Nages (Gard) sont reliés à la période couvrant le début de La Tène C2 jusqu'à la fin de La Tène D1a (Feugère et Py, 1989). Pour une attribution allant du début de La Tène $\mathrm{C} 2$ jusqu'à 50 de notre ère, des comparaisons sont également possibles avec les exemplaires du " Gué-de-Sciaux » à Antigny (Vienne) (Bertrand, 2007).

- Type 2C2I.b. (tabl. 4, XVIII). Ce bracelet bleu, à double cordon incisé transversalement et encadré par de simples cordons, a été mis au jour au nord du cairn, au cours de la fouille de la tombe mégalithique de "Beaumont » à SaintLaurent-sur-Oust (Morbihan) (Tinevez et al., 1990, p. 46). Lauteur a daté $\mathrm{du} \mathrm{I} \mathrm{I}^{\mathrm{er}}$ siècle avant notre ère ce bracelet (scindé en deux fragments), d'après le mobilier (des fragments d'amphore de type Dressel 1) trouvé à cet endroit et d'après les travaux d'Haevernick et Gebhard.

Des bracelets analogues sont connus à La Tène $\mathrm{C} 1$ dans le canton de Fribourg (Suisse) et à Manching en Allemagne (Ramseyer, 1995; Gebhard, 1989b). D'autres bracelets bleus de ce type sont associés à un contexte du début La Tène C1 jusqu'à la fin de La Tène D1 sur le site de Nage (Gard) (Feugère et Py, 1989).

- Type 2C3B.b (tabl. 4, XIX). Ces bracelets bleus présentent des séquences de trois bourgeons sur un corps central encadré par deux fins cordons. Ils proviennent du dépôt de la Lande du Rameau ( ${ }^{\circ} 4$ ) à Brec'h (Morbihan) et de l'habitat des Ebihens ( $\left.\mathrm{n}^{\circ} 1\right)$ à Saint-Jacut-de-la-Mer dans les Côtes-d'Armor. Avec une couleur différente (incolore avec une couche interne jaune) ce type bourgeonnant est daté de La Tène C2 à la fin de La Tène D2 sur le site de Nage (Feugère et Py, 1989).

- Type 2C4B.b (tabl. 4, XX). Ce bracelet bleu cobalt montre une séquence de quatre bourgeons (le quatrième étant un peu aplati), sur un corps central encadré par deux fins cordons. Sur le site de "La Ville Pollo " à Trégueux (Côtes-d'Armor), il est associé à un ensemble du début de La Tène D (Dinard, 2008), de même que son homologue mis au jour à Epiais-Rhus dans le Val-d'Oise (Vanpeene, 1989).

- Type 4CT.b (tabl. 4, XXI). De couleur bleu cobalt, notre seul représentant de ce type est constitué de cordons obliques (séparés par des sillons ponctués réalisés au peigne), imitant une torsade et bordés par des cordons simples; il provient des Ebihens à Saint-Jacut-de-la-Mer dans les Côtesd'Armor (bracelet 3).

Ce type est attribué du début de La Tène $\mathrm{C} 1$ jusqu’à la fin de La Tène D2 à Nage dans le Gard (Feugère et Py, 1989). Sur le site de Mars Mullo à Allonnes (Sarthe), il est connu à La Tène C (Brouquier-Reddé et Gruel, 2004), alors qu'à Mandeure (Doubs), il est daté du début de La Tène C2 jusqu'à La Tène D2 (Guillard, 1989). Ce bracelet bleu trouve également des références à La Tène $\mathrm{C} 2$ au Village des Arènes à Levroux dans l'Indre (Tilliard, 1989). 
- Type 4CT.b.zy*zw (tabl. 4, XXII). Ce bracelet bleu cobalt se compose de cordons obliques imitant une torsade, encadrés de part et d'autre par deux fins cordons. Des zigzags, dont un jaune, décorent le cordon torsadé. Notre unique exemplaire est issu du site d'habitat de Kerhilio $\left(\mathrm{n}^{\circ} 4\right)$ à Erdeven (Morbihan), où il est associé à La Tène $\mathrm{D}$ (Jacq, 1941.

Il en est de même pour celui d'Epiais-Rhus dans le Vald'Oise (Vanpeene, 1989). Mais, sur le site de La Vache à l'Aise à Bobigny (Seine-Saint-Denis), ce type de bracelet est daté de La Tène C (Le Béchennec et al., 2005; phase II), tout comme dans le canton de Fribourg en Suisse (Ramseyer, 1995). Enfin, un bracelet bleu de même coupe et ornementation est attribué à un milieu daté de La Tène C et La Tène D à Nage dans le Gard (Feugère et Py, 1989).

- Type 4CV (tabl. 4, XXIII). Notre seul bracelet de ce type est bleu et montre deux cordons simples et fins encadrant de chaque côté un large cordon central marqué par des chevrons réalisés au peigne. Il a également été mis au jour à «Kerhilio " $\left(\mathrm{n}^{\circ} 3\right)$ et, comme le précédent, a été daté de La Tène D (Jacq, 1941). Un bracelet de Besançon ayant des chevrons apposés sur cinq cordons (5C/V.b dans notre code) est daté de La Tène D (Bride, 1999). Cette datation coïncide avec celle de Kerhilio même si les bracelets diffèrent légèrement.

- Type 4CV/T.b.zy zw (tabl. 4, XXIV). De couleur bleue, ce bracelet se compose de deux cordons simples et fins, de part et d'autre d'un cordon épais. Ce cordon central présente des chevrons tout en étant torsadé, les chevrons étant décorés de zigzags blancs et jaunes. Il s'agit du $\mathrm{n}^{\circ} 3$ du dépôt de la Lande du Rameau à Brec'h (Morbihan). On lui trouve des références à la transition La Tène C2/La Tène D1 en Tchécoslovaquie (Venclovà, 1989). Sur le site de Nage, ce type de bracelet (mais incolore à fond jaune) est associé à La Tène D1b (Feugère et Py, 1989).

\section{En résumé...}

La typologie ainsi mise en place a permis d'élargir les comparaisons et d'apporter des éléments de datation pour tous les bracelets étudiés (tabl. 4). On a pu constater des variantes chronologiques au sein d'un même groupe typologique; ceci est peut-être en corrélation avec le mode de production et la nature des composants du verre employé. Ces variantes peuvent aussi s'expliquer par le fait que des objets de comparaison proviennent de sites parfois très éloignés de la Bretagne. Il convient donc, pour ces derniers, de prendre en compte leur apport typochronologique avec pondération.

\section{Analyses ChIMiQues}

\section{Protocole}

Le bracelet de "l'Homme Mort » a été analysé par microsonde électronique, sous la direction de Nathalie Brun, au laboratoire de Recherche des Musées de France (Palais du Louvre). Seize autres ont été analysés par spectrométrie de masse à plasma avec prélèvement par ablation laser (LA-ICP-MS) au laboratoire du centre Ernest-Babelon (IRAMAT, UMR 5060 du CNRS/Université d'Orléans), par Bernard Gratuze (fig. 2).

Cette dernière méthode est particulièrement bien adaptée aux objets composites ou de petite taille comme les perles et les fragments de bracelets. Lors de l'analyse, les objets sont placés à l'intérieur d'une cellule en quartz (fig. 3). Un micro-prélèvement, invisible à l'œil nu, est

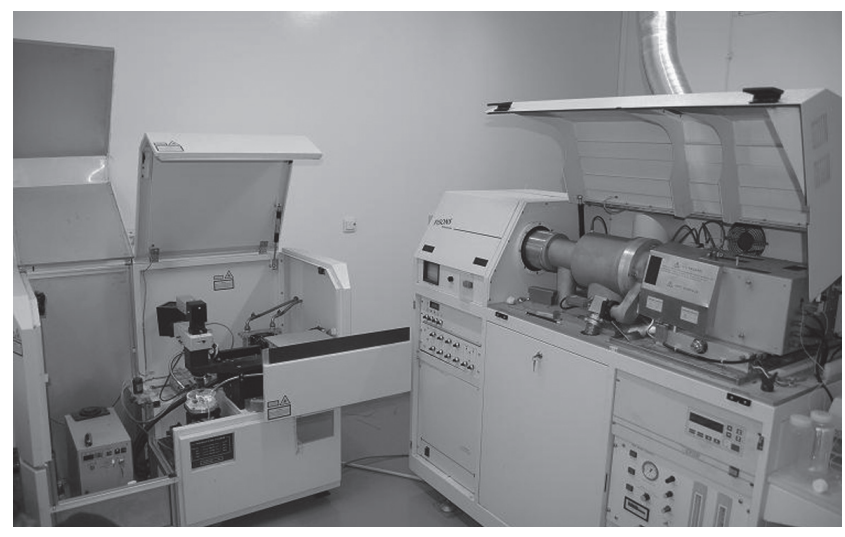

Figure 2 : Vue de l'appareil ICP-MS utilisé pour l'analyse des verres. Figure 2: View of the ICP-MS equipment used for glass analysis.

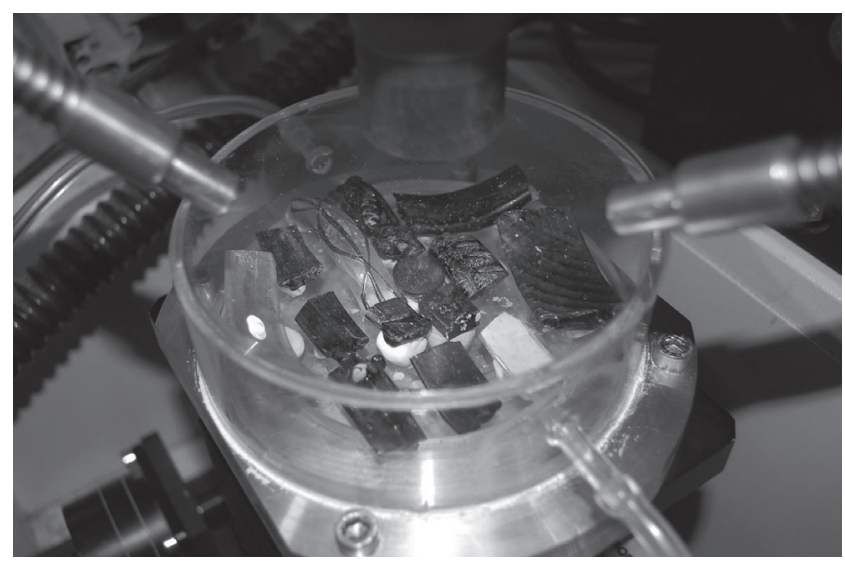

Figure 3 : La cellule en quartz renfermant les fragments de bracelets en cours d'analyse.

Figure 3: The quartz cell containing the bracelets fragments to be analysed. 
effectué par un rayon laser (diamètre 80 micromètres, profondeur 200 micromètres). La matière prélevée (quelques microgrammes) est transportée vers une torche à plasma par un flux gazeux d'argon. La haute température du plasma $\left(8000^{\circ} \mathrm{C}\right)$ dissocie et ionise la matière, dont les différents constituants sont identifiés selon leur masse. Un détecteur électronique permet leur quantification. L'étude des objets est réalisée sans aucune préparation de l'échantillon. Pour chaque analyse, de quatre à six prélèvements (deux ou trois pour les éléments majeurs et deux ou trois pour les éléments mineurs et les traces) sont effectués à un endroit sain de la surface de l'objet. Le calcul est effectué sur la moyenne des prélèvements. L'étalonnage est effectué à l'aide des verres étalons développés par le NIST ${ }^{3}$ (SRM610) et la Corning ${ }^{4}$ (verres A, B, C et D) ainsi que par des verres archéologiques de composition connue.

\section{Résultats des analyses}

Le verre est un matériau composite (Gratuze, 1994). Le mélange de base est constitué d'un vitrifiant (la silice, provenant du sable, de galets de quartz, ou encore de verre de récupération), d'un fondant (soude ou potasse par exemple) pour abaisser la température de fusion de l'élément vitrifiant, et d'un stabilisant (chaux ou alumine) pour éviter que le mélange ne soit soluble ${ }^{5}$. A ce mélange, le verrier peut ajouter des agents opacifiants (l'antimoniate de calcium pour le blanc, l'antimoniate de plomb pour le jaune) et des agents colorants, sels ou oxydes de métaux tels que le manganèse pour le violet et l'incolore, le cobalt pour le bleu, le fer pour le vert et le brun ambré, le cuivre pour le bleu et le vert. Tous ces ingrédients sont généralement accompagnés d'un cortège d'impuretés qui peuvent servir de traceurs pour suivre la fabrication du verre; ce sont donc des éléments non négligeables.

Tous les bracelets n'ont pas pu être analysés. Mais ceux qui l'ont été (17 sur 43), ont apporté des éléments intéressants. En effet, en plus de pouvoir déterminer les familles chimiques auxquelles appartiennent les verres, on a pu émettre des hypothèses sur les régions de production de la matière première utilisée. D'après les analyses, on distingue deux groupes de verres dans notre série de bracelets.

3. le NIST (National Institute of Standards and Technology) est une agence américaine tendant à promouvoir l'économie via l'amélioration et la recherche dans divers domaines scientifiques et industriels [www. nist.gov].

4. Corning est une société américaine spécialisée dans le verre et la céramique servant à la science et plus particulièrement la médecine, la technologie et les télécommunications [www.corning.com].

5. L'ajout d'un fondant provoque la rupture d'une liaison Si-0 et l'apparition d'un oxygène libre. Ceci a pour conséquence de fragiliser le réseau atomique et d'augmenter la solubilité à l'eau.
- Le premier groupe est constitué des verres bleus de Trégueux, Mordelles et Saint-Laurent-sur-Oust, ainsi que des verres verts de Landeda, Quéménéven, Erdeven (bracelets 1 et 2) et Saint-Nicolas-du-Pelem, du verre incolore de Mordelles et des verres jaunes utilisés pour les décors des bracelets de Plourin-Ploudalmezeau, d'Erdeven $\left(\mathrm{n}^{\circ} 4\right)$ et d'Arzon.

- Le second groupe est représenté par les verres bleus du Quiou, de Saint-Pierre-de-Plesguen, de PlourinPloudalmezeau, d'Erdeven (bracelets 3 et 4) et d'Arzon, le verre vert de Lamballe, le verre brun de Paule, le verre incolore et le verre jaune servant à la couche interne du bracelet de Ploufragan, ainsi que par le verre blanc du décor d'Arzon.

Selon B. Gratuze (communication orale), ces deux groupes de verres seraient originaires d'ateliers primaires distincts, mais situés tous deux au Proche-Orient. Notons que les verres bleus des bracelets d'Erdeven $n^{\circ} 4$, d'Arzon et de Plourin-Ploudalmezeau paraissent avoir été produits dans des ateliers primaires différents de celui qui a produit le verre jaune des décors de zigzags qu'ils arborent.

\section{DisCusSION ET CONCLUSION}

Le codage typologique et les analyses chimiques appliqués sur les bracelets protohistoriques en verre de la Péninsule armoricaine nous ont permis d'effectuer de multiples observations.

Tout d'abord, des corrélations entre typologie et matière première ne sont pas encore véritablement possibles, puisqu'il y a trop peu de bracelets analysés au sein de chaque type. Mais le codage a facilité la datation des bracelets bretons ainsi que les comparaisons avec d'autres bracelets (de France et d'ailleurs). Ainsi, pouvons-nous constater que les verres verts datés de La Tène $\mathrm{D}$, qui sont colorés par le Fer (et correspondent à des pièces de types I et $\mathrm{T}$ ), proviennent d'un même centre de production; c'est le cas des bracelets 1 et 2 d'Erdeven et de celui de Quéménéven. En revanche, les verres verts et bleus de la même époque, mais colorés par le cobalt et le cuivre (Saint-Nicolas-du-Pelem et Lamballe) peuvent provenir de centres de production différents.

Les verres des bracelets de type 3C qui ont été étudiés et analysés s'avèrent être propres à un atelier primaire de production du verre; c'est le cas des parures de Landeda et Saint-Nicolas-du-Pelem. Mais il faudrait pouvoir analyser d'autres bracelets de même typologie pour pouvoir confirmer ou infirmer l'importance de cette donnée.

En ce qui concerne les verres jaunes des bracelets bretons, les analyses ont révélé que les décors jaunes semblaient provenir d'un atelier primaire différent de celui ayant produit le verre jaune destiné à la couche interne. Là encore, il 
faudrait pouvoir analyser de nombreux autres verres jaunes pour généraliser ce propos; en attendant, des analyses sur des perles armoricaines tendent à renforcer cette hypothèse (communication de B. Gratuze).

Ensuite, l'absence apparente de relation entre la typochronologie des bracelets et la provenance de la matière première montre qu'il ne paraît pas y avoir de variations ni de préférences d'approvisionnement en verre au cours du second âge du Fer. Par exemple, les colorations des bracelets au cobalt et à l'antimoniate de plomb ne sont pas spécifiques à un centre de production.

Il ne semble pas non plus y avoir de distribution préférentielle de ces parures par rapport à la fonction du site. En effet, on a mis au jour quasiment autant de bracelets en verre sur des habitats (10 sites) que sur des sites à fonction funéraire et/ou cultuelle (7 sites). Mais de nombreux bracelets ont été découverts lors de prospections et sont de ce fait hors contexte.

Les diverses méthodes employées ont apporté de précieux résultats mais ne sont pas exemptes de limites. Tout d'abord, notre proposition de code typologique n'est qu'une ébauche et nécessiterait d'être approfondie pour être plus opérationnelle.

Quant aux analyses chimiques, elles ne renseignent que sur la matière première. Nous pouvons certes proposer des zones différentes de productions des verres, mais les objets ont pu être élaborés ailleurs, dans des ateliers secondaires. $\mathrm{Ni}$ la typologie ni les analyses ne permettent à ce jour de faire la distinction entre ateliers primaires et secondaires, ni même de distinguer un premier emploi de produits semifinis de refontes d'objets déjà élaborés. En plus, il est possible qu'au sein des ateliers secondaires, il y ait eu un mélange de verres d'origines géographiques différentes. Par exemple, si certains bracelets ont été fabriqués en Gaule à partir de verre brut importé, les artisans gaulois ont pu utiliser des verres produits à différents endroits pour la production d'un même objet. Comme l'a soulevé Bernard Gratuze (Gratuze et Marchetti, 2007), ce possible mélange de verres implique une multiplication des types chimiques et rend la détermination des ateliers primaires plus difficile.

Une autre limite de ces méthodes (typologie et analyse par spectrométrie de masse) est qu'elles ne permettent pas de comprendre ni de définir la façon dont les bracelets ont été mis en forme : ont-il été moulés? Ont-ils été formés à partir des mouvements simultanés de deux (ou plusieurs) baguettes? Ont-ils été coupés à partir d'une masse de verre enroulée sur un rondin de bois? Etc.

Par la double origine des verres des bracelets d'Erdeven $\mathrm{n}^{\circ} 4$, d'Arzon et de Plourin-Ploudalmezeau, nous avons mis en relief l'existence d'un réseau de distribution complexe du verre (sous forme de produits finis et/ou semi-finis). Les bracelets bretons confirment ainsi l'existence de relations entre le nord-ouest de la France et les pays méditerranéens telle qu'elle a pu être pressentie à propos des perles en verre par B. Gratuze (communication orale).

Nous pouvons aussi remarquer une inégalité de distribution des bracelets découverts sur le territoire breton : il y en a beaucoup plus sur le littoral qu'à l'intérieur des terres. Cette inégalité est-elle due aux stratégies de fouille et de prospection ou est-elle liée aux modes de transport et d'échanges (rôle des voies maritimes par exemple)?

L'application du codage typologique et de l'analyse chimique à d'autres bracelets en verre est indispensable pour répondre aux problématiques soulevées par cette étude (notamment en ce qui concerne l'établissement de liens entre la typologie, la datation et la composition des bracelets). Il serait de même intéressant d'établir des parallèles morphologiques et iconographiques avec d'autres objets (perles en verre, bracelets en métal, etc.) et de trouver un moyen d'étude nous permettant de répondre aux questions de détermination géographique des ateliers secondaires et de détermination du mode de fabrication des bracelets.

\section{Remerciements}

Je tiens à remercier l'équipe du laboratoire Centre ErnestBabelon - IRAMAT; Gilles Leroux, Serge Mentele et Laurent Beuchet (responsables d'opération à l'INRAP); Laure Simon et Françoise Labaune (céramologues à l'INRAP); Vincent Pommier (topographe à l'INRAP); Yves Menez, Anne Villard-Le-Tiec, Emile Bernard et Jean-Yves Tinevez (SRA de Bretagne); Alain Triste, du CERAM; Michel Le Goffic et le SDA du Finistère; Catherine Bizien-Jaglin et le CERAA; Jean-Laurent Monnier et le Musée de Préhistoire finistérienne à Penmarc'h; Françoise Berretrot et le Musée de Bretagne à Rennes; Emmanuelle Vigier et le Musée de la Préhistoire à Carnac; Christophe Lepennec et le Musée de Vannes; Catherine Petit-Aupert, Maître de Conférence à l'université Rennes 2 et Marie-Yvane Daire, Chargée de Recherche au CNRS (UMR 6566).

Toutes ces personnes mont aidée à la constitution de l'inventaire des bracelets bretons et certaines ont même facilité leur prêt en vue des analyses. Je suis également redevable de nombreuses illustrations: Mme Labaune pour le bracelet du Quiou au code 2C2T (tabl. 4, XVII); M. Menez (le Boisanne au code 2C: tabl. 4, VIII); M. Langouet (le $n^{\circ} 1$ des Ebihens au code 4CT.b : tabl. 4, XXI); Mme Simon (Bilaire au code 3C.b et Ploufragan au code 5C.iny: tabl. 4, IX et XIII); M. Galliou (la Boissière au code T.g : tabl. 4, XV); M. Leroux (l'Homme Mort au code D.b : tabl. 4, I); M. Batt (Sermon au code D.b.3fb : tabl. 4, VII). 


\section{Bibliographie}

Bataille, G., 2008 - Les Celtes : des mobiliers aux cultes. Dijon, Editions Universitaire de Dijon, 258 p.

BATT, M., 1987 - Mordelles (35) Sermon : site gaulois et temple celto-romain (rapport de sauvetage programmé). Rennes, SRA de Bretagne, 30 p., 21 fig., multigraphié.

Bertrand, I., 2007 - Les parures en métal, en lignite et en verre liées à l'occupation de La Tène finale dans le sanctuaire galloromain du Gué-de-Sciaux (Antigny, Vienne), in Duval, A. et Gomez de Soto, J. (dir.), Sites et mobiliers de l'âge du Fer entre Loire et Dordogne. Chauvigny, Association de Publication Chauvinoise (mémoire XXIX), p. 78-85.

Bizien-Jaglin, C., 1992 - L'îlot des Haches, Saint-Jacut-de-laMer (Côtes d'Armor), bilan de trois campagne de fouille. Les Dossiers du Centre de Recherche archéologique d'Alet, $\mathrm{n}^{\circ} 20$, p. 119-123.

Bizien-Jaglin, C., Galliou, P. et Kérébel, H., 2003 - Carte archéologique de la Gaule : pré-inventaire archéologique des Côtes d'Armor (22). Paris, Académie des Inscriptions et BellesLettres, $406 \mathrm{p}$.

Bizien-Jaglin, C. (dir.), 2004 - Les Haches en Saint-Jacut-de-laMer (Côtes d'Armor). Saint-Malo, Les Dossiers du Centre de Recherche archéologique d'Alet, supplément AA, 167 p.

BousqueT, J., 1961 - Informations archéologiques, circonscription de Rennes. Gallia, 19 - 2, p. 345-354.

Briard, J., Giot, P.-R. et Pape, L., 1995 - Protohistoire de la Bretagne, Rennes, Ouest-France université, 423 p. ( $2^{\mathrm{e}}$ édition).

BRIDE, A.-S., 1999 - La parure celtique au deuxième âge du Fer dans l'Est de la Gaule. Bulletin de l'Association française pour l'Archéologie du Verre, ${ }^{\circ}$ 7, p. 3-4.

BRIDE, A.-S., 2005 - Le mobilier de verre des fouilles anciennes et récentes de Bibracte, in Guillaumet, J.-P. et Szabo, M. (dir.), Études sur Bibracte, Bibracte, n 10 - 1, p. 81-96.

Brouquier-Redde, V. et Gruel, K., 2004 - Le sanctuaire de Mars Mullo, Allonnes (Sarthe).Le Mans, Editions de la Reinette, 190 p.

Clément, M. et Galliou, P., 1985 - Le dépôt gaulois de Brec'h (Morbihan). Revue Archéologique de l'Ouest, 2, p. 65-71.

Dinard, M., 2008 - Le mobilier protohistorique de Trégueux, in Leroux, G., Desfonds, A., Labaune-Jean, F. et Freita, J., Rocade d'agglomération de Saint-Brieuc (Bretagne, GrandOuest) : Tregueux, section "La Crarée-Le Perray" (22). Rennes, INRAP, SRA de Bretagne et Conseil général des Côtesd'Armor, 88 p., 20 fig., multigraphié.

DinARD, M., 2009 - Les bracelets protohistoriques en verre de la Péninsule Armoricaine (mémoire de Master 2), Rennes, Université Rennes 1, 81 p.

Feugère, M. et Py, M., 1989 - Les bracelets en verre de Nages (Gard), in Feugère, M. (dir.), Le verre préromain en Europe occidentale, Montagnac, éd. Monique Mergoil, p. 153-167.
GALliou, P., 1982 - Glanes archéologiques, in (collectif) La villa romaine de La Boissière en Lanvéoc (Finistère), Archéologie en Bretagne, $\mathrm{n}^{\circ} 33-34$, p. 19-31.

Galliou, P., 1989 - Carte archéologique de la Gaule : pré-inventaire archéologique du Finistère (29). Paris, Académie des Inscriptions et Belles-Lettres, $230 \mathrm{p}$.

Gebhard, R., 1989a - Pour une nouvelle typologie des bracelets celtiques en verre, in FEUGÈRE, M. (dir.), Le verre préromain en Europe occidentale, Montagnac, éd. Monique Mergoil, p. 73-83.

GEbHaRd, R., 1989b - Le verre à Manching : données chronologiques et apport des analyses, in Feugère, M. (dir.), Le verre préromain en Europe occidentale, Montagnac, éd. Monique Mergoil, p. 99-106.

Gratuze, B., 1994 - Le verre : problématiques posées et réponses apportées par les méthodes de caractérisation physico-chimiques, Revue d'Archéométrie, 18, p. 75-87.

Gratuze, B. et Marchetti, M.-L., 2007 - Le mobilier en verre du site de la Grotta Piatta (Aregno, Haute-Corse) : composition chimique et chronotypologie, Archéosciences - Revue d'Archéométrie, 31, p. 163-173.

GuILlard, M.-C., 1989 - La verrerie protohistorique de Mandeure (Doubs), in Feugère, M. (dir.), Le verre préromain en Europe occidentale, Montagnac, éd. Monique Mergoil, p. 145-152.

Haevernick, T.-E., 1960 - Die Glasarmringe und Ringperlen des Mittel- und Spätlatènezeit auf dem europäischen Festland. Bonn, Rudolf Habelt Verlag, 272 p., 35 pl.

JaCQ, M., 1941 - Catalogue du musée archéologique James Miln Zacharie Le Rouzic, Vannes, Lafolye et Lamarzelle, 258 p.

Kaenel, G. et Müller, F., 1989 - À propos de certains types de bracelets en verre du Plateau suisse, in Feugère, M. (dir.), Le verre préromain en Europe occidentale, Montagnac, éd. Monique Mergoil, p. 121-128.

Langouët, L., Bucur, I., Daire, M.-Y., Forrières, C., Giot, P.-R., Krausz, S., Marguerie, D. et Morzadec, H., 1989 Un village coriosolite sur l'île des Ebihens (Saint-Jacut-de-la-Mer). Saint-Malo, Les Dossiers du Centre de Recherche archéologique d'Alet, supplément L, 173 p.

Labaune, F. et Petit-Aupert, C., 2005 - Les campagnes antiques aux confins des cités des Coriosolites et des Riedones : le cas du Bassin du Quiou et de sa périphérie (Ille-et-Vilaine et Côtes d'Armor), rapport de campagne de prospection. Rennes, SRA de Bretagne, 17 p., multigraphié.

Le Bechennec, Y., Marion, S. et Metrot, P., 2005 - L'occupation protohistorique de Bobigny (Seine-Saint-Denis), in Buchsenschutz, O., Bulard, A. et Lejars, T. (dir.), L'àge du Fer en Ille-de-France (Actes du 26 colloque AFEAF, ParisSaint-Denis, 2002). Revue archéologique du Centre de la France, supplément $n^{\circ} 26$, p. 97-126.

Le Bechennec, Y., Le Forestier, C. et Marion, S., 2007 Nécropole et bourgade d'artisans : l'évolution des sites de 
Bobigny (Seine-Saint-Denis), entre La Tène B et La Tène D. Revue archéologique du Centre de la France, 45-46 [http://racf. revues.org/index654.html].

LE Bihan, J.-P., 2007 - Vestiges protohistoriques et antiques découverts à Mez-Notariou (Ouessant) (rapport intermédiaire de fouille programmée). Rennes, SRA de Bretagne/Quimper, CRAF, Conseil Général du Finistère et SDA du Finistère, 40 p., 45 fig., multigraphié.

Lecornec, J., 1994 - Le Petit Mont, Arzon (Morbihan). Rennes, RAO (coll. Documents archéologiques de l'Ouest), 109 p.

Le Goffic, M. et Peuziat, J., 1997 - Quéménéven, Kerfloux, in Galliou, P. (dir.), Notices d'archéologie finistérienne (année 1996). Bulletin de la Société archéologique du Finistère, 126, p. 33-77.

Leroux, G., Brun, N., Daire, M.-Y., Guillard, M.-C., Langouët, L., Le Page, G. et Morzadec, H., 1991 - Un établissement gaulois de Haute-Bretagne : la fouille du site de L'Homme Mort en Saint-Pierre-de-Plesguen (Ille-et-Vilaine). Saint-Malo, Les Dossiers du Centre de Recherche archéologique d'Alet, supplément N, 90 p.

Menez, Y., 1996 - Une ferme de l'Armorique gauloise : le Boisanne à Plouër-sur-Rance (Côtes d'Armor), Paris, Maison des Sciences de l'Homme, coll. « Documents d'Archéologie Française; 58 », $270 \mathrm{p}$.

Menez, Y., 2008 - Le Camp de Saint-Symphorien à Paule (Côtes d'Armor) et les résidences de l'aristocratie du second âge du Fer en France septentrionale (Thèse), Paris, Université Paris I (Panthéon-Sorbonne), 559 p., 548 fig.

MiLn, J., 1882. Explorations du Mané-Roullarde, auprès de La Trinité-sur-Mer (Morbihan). Bulletin de la Société polymathique du Morbihan, 26, p. 70-77.

Pré, S. et Galliou, P., 2000 - Quéménéven, kerfloux, in Galliou, P. (dir.) - Notices d'Archéologie finistérienne (1999-2000). Bulletin de la Société archéologique du Finistère, 129, p. 23-43.
RAMSEYER, D., 1995 - Trésors des Celtes : archéologie dans le canton de Fribourg (Suisse), Treignes, Cedarc, $47 \mathrm{p}$.

Robinson, C., 2003 - Size does not matter: the nature of celtic bead-making sites. Ornaments from the past : bead studies after beck, a book on glass and semiprecious stone beads in history and archaeology for archaeologists, jewellery historians and collectors, London, The Bead Study Trust, p. 79-91.

SANQUER, R., 1975 - Informations archéologiques, circonscription de Bretagne. Gallia, 33-2, p. 346-347.

SchWAB, H., 1995 - Deux nécropoles laténiennes à Gumefens (canton de Fribourg, Suisse), in Charpy, J.-J. (dir.), L'Europe celtique du ve au III siècle avant J.-C. : contacts, échanges et mouvements de populations (Actes du $2^{\mathrm{e}}$ symposium international d'Hautvilliers, Epernay 1992). Sceaux, Kronos B. Y. éditions (Mémoire $\mathrm{n}^{\circ} 9$ de la Société archéologique champenoise), p. 239-259.

Simon, L., 2001 - Les mobiliers, in Baillieu, M. (dir.), Vannes : le sanctuaire gallo-romain de Bilaire (Morbihan) (DFS de fouille programmée 1999-2000), Rennes, AFAN et SRA de Bretagne ( $2^{\mathrm{e}}$ volume), 83 p., multigraphié.

Tilliard, L, 1989 - Les bracelets et les perles en verre de Levroux (Indre), in Feugère, M. (dir.), Le verre préromain en Europe occidentale, Montagnac, éd. Monique Mergoil, p. 138-144.

Tinevez, J.-Y., Baud, C.-A., Grévin, G., Lagier, R., Giot, P.-R. et MorZadec, H., 1990 - La sépulture mégalithique à entrée latérale de Beaumont à Saint-Laurent-sur-Oust (Morbihan) : études anthropologique et pétrographique, données complémentaires, Revue archéologique de l'Ouest, 7, p. 41-56.

VANPEENE, N., 1989 - Le verre préromain à Epiais-Rhus, in FEUGÈRE, M. (dir.), Le verre préromain en Europe occidentale, Montagnac, éd. Monique Mergoil, p. 129-136.

Venclova, N., 1989 - Parure celtique en verre en Europe centrale, in Feugère, M. (dir.), Le verre préromain en Europe occidentale, Montagnac, éd. Monique Mergoil, p. 84-97. 